\title{
CASE EXAMINERS - A PANACEA OR A CURSE?
}

The BDJ Upfront section includes editorials, letters, news, book reviews and interviews. Please direct your correspondence to the News Editor, Kate Quinlan at the BDJ, The Macmillan Building, 4 Crinan Street, London, N1 9XW or by email to BDJNews@nature.com

Press releases or articles may be edited, and should include a colour photograph if possible.

Peter Ward, Chief Executive BDA

$\mathrm{T}$ he consultation on the implementation of the amendments to the working practices of the GDC is now open. Central to those amendments is the creation of a team of case examiners who will be empowered to fast track concerns raised with a view to avoiding formal fitness to practise proceedings.

In principle, the BDA has expressed its support for any moves to make the process more efficient and reduce the stresses attached with it. Consequently, it is our belief that the profession should give these proposals very thorough consideration. As always, the devil is in the detail. And the significance of how the scheme is implemented is so profound as to render the changes either a massive improvement or a significant threat.

First the case for the massive improvement: imagine a team of highly intelligent, pragmatic and balanced case examiners properly empowered to make reasonable decisions and to filter the wheat from the chaff. Imagine the range of outcomes available to those case examiners to be truly performance enhancing and offering genuine opportunities for professionals to reflect on areas of their practice and to raise their game. Imagine a regime that promoted a culture where performance was improved in a spirit of candour and in the absence of blame and recrimination. Immediately, the implementation of this scheme would help professionals improve the quality of care, it would reduce the wasteful costs of trumped up cases, it would be better for patient protection and would substantially reduce levels of practitioner stress.

So what is there to be scared of? Well in the reassuring world I have described above there are quite a few expectations. But for that to turn

\section{...how the changes are imple- mented could be a massive improve- ment or a significant threat...'}

into reality we need to consider the risks buried within the people and the practices at work, and also in the products of their labours.

The GDC will need to clearly demonstrate that it has selected carefully and that its systems hold up to scrutiny. Additionally, the profession will need to be reassured that the GDC Council is properly overseeing the practices and intervening when necessary. It is to be hoped that the recent criticisms and undertakings made by the Council represent a new beginning and one where the GDC can be trusted to get it right.

The consultation explores how the GDC might agree undertakings with practitioners and what warnings it might be able to issue. Here lie the most substantial threats. If practitioners do nothing else they should express their views on this consultation since case examiners are to have enormous discretion and will decide upon the frame of words that are applied to the registrant's record. The details contained in those words are anticipated to be much more graphic than the summary determinations currently published after formal hearings. Disturbingly, it would also appear that those words will remain on the registrant's record in perpetuity.

So prospective 'defendants' face a choice. They can undergo the lengthy and painful process of formal investigation with the risk of sanctions. Statistically the historic likelihood of falling foul of sanctions in that process is actually very small (in 2015 from approximately $100,000+$ registrants fewer than 400 were found to have acted in a way warranting some kind of sanction) and for the vast majority entering that process who suffered no sanction there is no blemish on their record, enabling them to continue in practice with their reputations intact.

It is likely that the enticement of early disposal of a case may well appeal to many under the new arrangements, but any satisfaction may be short lived if it results in a permanent blemish on the registrant's record. And what of the purpose here? If we are trying to encourage openness and reflection, isn't that rather compromised if the respondent is then to suffer an indelible mark on their reputation? As proposed, the note on the record looks more like punishment and humiliation than a serious encouragement to performance improvement. So we do need some clarity here before we face the prospect of a sizeably larger number of registrants with adverse comments attached to them?

With this situation pertaining one wonders whether the smart money might militate against accepting this fast track process altogether. Better to take a risk and enjoy the $90+\%$ chance that one will escape invisible and untarnished rather than sign up to an agreed public statement of your own suggested inadequacy?

This matter is a serious one. It is central to whether the anticipated cost savings will be delivered. Creating a system whereby the reputational damage ensuing is greater than the formal process will do nothing to drive uptake. So, in the new world of a GDC that says it is listening, please make your views heard. There is real opportunity in these proposals, but only if the difficult and significant by-products are fully evaluated. The consultation is open until 14 March 2016 to have your say. Read. Think. Tell 'em.

DOI: 10.1038/sj.bdj.2016.109 